\title{
Linear Optimal Multi-periodic Repetitive Control -A Low Order Controller Scheme
}

\author{
Youde $\mathrm{Han}^{a}$, David H. Owens ${ }^{b}$, and Bing $\mathrm{Chu}^{c}$ \\ ${ }^{a}$ Department of Automatic Control and Systems Engineering, \\ University of Sheffield, Sheffield, United Kingdom, S1 3JD \\ Email: youdehan@yahoo.com \\ ${ }^{b}$ Department of Automatic Control and Systems Engineering, \\ University of Sheffield, Sheffield, United Kingdom, S1 3JD \\ Email: d.h.owens@shef.ac.uk \\ ${ }^{c}$ Department of Engineering Science, University of Oxford \\ Parks Road, Oxford, United Kingdom, OX1 3PJ \\ Email: bing.chu@eng.ox.ac.uk
}

(Received October 31, 2011; in final form December 28, 2011)

\begin{abstract}
This article proposes a new method for treating the computational problem in discrete time linear multi-periodic repetitive control systems, where the reference signal includes several periodic components with already known periods. As periods get large, the computational problem becomes prominent. This work thus investigates the frequency contents of reference signals, where the order of original repetitive controller is lowered by considering only a reduced number of poles, namely, the most important contributors to the total energy of the multi-periodic reference signal. A lower order multi-periodic repetitive controller is designed which assures BIBO stability of the closed-loop system, and approximate tracking is achieved. Finally, experimental tests on a non-minimum phase spring mass damper system demonstrate the effectiveness of this new controller.
\end{abstract}

Keywords: Optimal, Repetitive control, Computation, Multi-periodic, BIBO.

AMS Classification: 49N05, 65F10, 03D15, 49N20, 37J25

\section{Introduction}

Many signals associated with engineering are periodic, or at least they can be approximated by a periodic signal over a large time interval. This is true, in many tracking problems, the desired output or disturbance input is often composed of a periodic component with an already known period. In these cases, if one merely applies the standard feedback controllers such as PID, LQR or $H^{\infty}$ methods, the result will often have steady

Corresponding Author. Email: youdehan@yahoo.com. state tracking errors. In order to track or reject such periodic signals, internal model principle (IMP) [1] instead requires one to tailor the design by including a model of reference/disturbance signals in the controller and design the system for closed loop stability. In other words, perfect tracking in the steady state can be accomplished only if a generator of that signal is included in the stable closed-loop system. Such a generator is termed as an internal model and the controller method is termed as repetitive control or $R C$, which has been widely applied in rolling 
process [2], disc file servo system [3], robot manipulator [4], position control system [5], steel casting process $[6,7]$, magnet power supply [8] and many other areas $[9,10,11]$.

Of these works $[2,3,4,5,6,7,8,9,10,11]$, all have developed full order repetitive controller models based IMP, which is an essential requirement for perfect tracking. However, in many situations, including a full (or complete) order internal model is neither necessary nor computationally effective due to their practical requirements. In these circumstances, turn to find lower order approximate internal models have become an interesting area for RC study. Although they cannot yield perfect tracking but they often require much less computation effort and in many cases yield near perfect tracking results. Previous researches on this subject are not many but a few publications are given in $[12,13,14]$.

Hillerstrom's paper [14] is a representative work on low order repetitive controller design. Their design is based on finding and considering the dominating harmonics of disturbance signals while the non-dominating ones are unconsidered. In their work, it is argued that sensitivity depends more on pole locations, with the poles more clustered, sensitivity becomes larger. It also mentioned that poles with larger number of harmonics on unit circle cause less sensitivity issues. Moreover, to ease the oscillation problem caused by discarding the high frequency components, a low-pass filter is introduced in the feedback path of the model to reduce the oscillations. This lowpass filter together with a lower order repetitive model assures asymptotic perfect tracking.

More recently, it has come to our notice that Wang et al. [15] have proposed a repetitive predictive controller based on frequency decomposition method, where a frequency sampling filter model is used to select the dominant frequency models, and Laguerre functions are applied to design the repetitive predictive controller. Their method show clear evidence of reducing controller algorithm complexity.

The present work will also be based on frequency analysis but will be approached from a different point of view. A few notable differences between this paper and these of $[14,15]$ will be: 1 . Hillerstrom et al. illustrate that there are dominating and non-dominating frequency parts of a periodic signal. However, they did not set forward a proper method to detect and separate these different frequency components, but only related the sensitivity to pole locations. 2. As claimed in Remark 2 of [14], the disturbance model there was selected to exactly match the disturbance signals, so it showed asymptotic perfect tracking. In practical situations, however, the energy of disturbance signal might not be concentrated to only a few frequencies but spread over all the frequency interval. e.g. square signals or triangular signals. In these cases, a detection tool also has to be found. 3. [14] only considered rejecting single periodic disturbance signals while it did not deal with tracking reference signals and multiperiodic cases. This work is thus aimed at solving the above three problems. 4. Wang et al. [15] used a frequency sampling filter model to select the dominant frequency components and designed the controller based on Laguerre functions, while the present work will approach the high computational problem from energy analysis of reference signals and the controller design is to be based on classical optimal control.

This paper is organized as follows: Section 2 reviews the earlier work by [16] and defines the problem. In Section 3, energy analysis of periodic and multi-periodic reference signals are investigated, and a low order multi-periodic repetitive control (MPRC) controller is designed for tracking multi-periodic signals purposes, with minor mathematical modifications, this method can also be extended to reject multi-periodic disturbance signals. Section 4 applies this new low order controller to a non-minimum phase spring mass damper system to demonstrate the effectiveness of the new MPRC controller and the impact of choosing different number of dominating poles on ultimate tracking performance is also illustrated. Finally, Section 5 concludes this paper and possible further research work is directed.

\section{Background and problem definition}

As a starting point, consider a linear time invariant (LTI) discrete time SISO system:

$$
\begin{aligned}
x(t+1) & =A x(t)+B u(t), x(0)=x_{o} \\
y(t) & =C x(t)
\end{aligned}
$$

where $A \in \mathbf{R}^{n \times n}, B \in \mathbf{R}^{n \times 1}, C \in \mathbf{R}^{1 \times n}$ are state matrices of appropriate dimension. $x(\cdot) \in \mathbf{R}^{n}$, 
$u(\cdot) \in \mathbf{R}$ and $y(\cdot) \in \mathbf{R}$ are states, input and output respectively. Alternatively, (1) can also be represented by its transfer function expression as:

$$
Y(z)=G(z) U(z)
$$

where $G(z)$ is the transfer function model.

The reference signal $r(\cdot) \in \mathbf{R}^{\mathbf{1} \times \mathbf{n}}$ is a discrete time multi-periodic signal with a linear combination of periodic components with different fundamental frequencies $f_{j}=\frac{2 \pi}{N_{j} h}$ representing large periods of $N_{j}$ times the 'sampling period' $h$, or it can be expressed as a time series:

$$
\begin{array}{r}
r(t)=\sum_{j=1}^{M} r_{j}(t), \quad r_{j}(t)=r_{j}\left(t-N_{j}\right) \\
1 \leq j \leq M, t \geq 0
\end{array}
$$

where $N$ is defined as the total period (or sum of individual periods) and the connection between $N$ and its individual periods $N_{j}$ is met by:

$$
N=\sum_{j=1}^{j=M} N_{j}, 1 \leq j \leq M
$$

Define the "annihilating polynomial" $P\left(z^{-1}\right)(z$ is the complex variable) as below:

$$
P\left(z^{-1}\right)=\prod_{j=1}^{M}\left(1-z^{-N_{j}}\right)=1+\sum_{j=1}^{N} \alpha_{j} z^{-j}
$$

where $\alpha_{j}$ is the coefficient of "annihilating polynomial" $P\left(z^{-1}\right)$. If the linear forward shift operator $\Delta$ is defined by the operation on the sequence $f(t)_{-\infty<t<\infty}$ with $\Delta f(t)=f(t+1)$ and its inverse (backward shift operator) as $\Delta^{-1} f(t)=f(t-1)$, then for multi-periodic signals over $-\infty<t<\infty$, the following equation is held:

$$
P\left(\Delta^{-1}\right) r(t)=0
$$

The proof of (6) is simple so it is omitted here. It is noted that $P\left(z^{-1}\right)$ may not be the minimum "annihilating polynomial" but can be replaced by a minimal one if the integers $N_{j}$ are relatively prime. It can be obtained from any annihilating polynomial by replacing multiple roots with a single simple root.
Given $\Delta^{-1}$ being the backwards shift operator, for any time series signal $v(t)$, the following relation is held:

$$
\tilde{\nu}(t)=\left(P\left(\Delta^{-1}\right) \nu\right)(t)=\nu(t)+\sum_{j=1}^{N} \alpha_{j} \nu(t-j)
$$

In [16], Owens et al. applied an internal model for a 'typical' discrete time MPRC system, where the MPRC model is integrated into the original system by having it's $z$-transfer function as:

$$
\frac{U(z)}{E(z)}=\sum_{i=1}^{M} \frac{\alpha_{i}}{1-W(z) z^{-N_{i}}}
$$

$W(z)$ is a low-pass filter being an exact internal model by choosing its value as 1 but only an approximate internal model otherwise. $\alpha_{j}$ is the coefficient of the "annihilating polynomial" that will be defined in the sequel. A block diagram of this MPRC model is in Figure 1

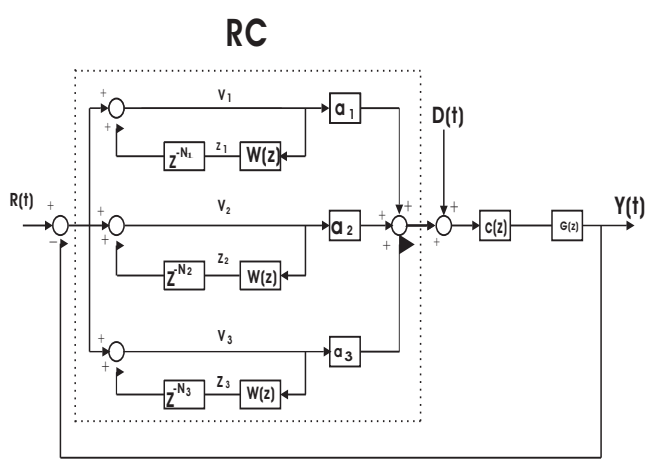

Figure 1. Owens' internal model

The main contribution of [16] is applying the condition (6) met by multi-periodic reference signal $r(t)$ to convert the original tracking problem between input controller $u(t)$ and output $y(t)$ into an LQR problem between a transformed controller $\tilde{u}(t)$ and error signal $e(t)$. By meeting the controllability and observability of this new representation, optimal control method was used for designing the MPRC law. After a mathematical manipulation, the augmented state-space representation derived in [16] has the following form: 


$$
\begin{aligned}
\underbrace{\left(\begin{array}{c}
\tilde{x}(t+1) \\
\Psi(t+1)
\end{array}\right)}_{Z(t+1)}=\underbrace{\left(\begin{array}{cc}
A & O \\
-F_{2} C & F_{1}
\end{array}\right)}_{\Pi} \underbrace{\left(\begin{array}{c}
\tilde{x}(t) \\
\Psi(t)
\end{array}\right)}_{Z(t)} \\
\quad+\underbrace{\left(\begin{array}{c}
B \\
O
\end{array}\right)}_{\Gamma} \tilde{u}(t) \\
e(t)=\underbrace{\left(\begin{array}{ll}
-C F_{3}
\end{array}\right)}_{\Omega} \underbrace{\left(\begin{array}{c}
\tilde{x}(t) \\
\Psi(t)
\end{array}\right)}_{Z(t)}(10)
\end{aligned}
$$

or simply as:

$$
\begin{aligned}
Z(t+1) & =\Pi Z(t)+\Gamma \tilde{u}(t) \\
e(t) & =\Omega Z(t)
\end{aligned}
$$

where $\Pi \in \mathbf{R}^{(n+N) \times(n+N)}, \Gamma \in \mathbf{R}^{(n+N) \times 1}$, $\Omega \in \mathbf{R}^{1 \times(n+N)}, Z(\cdot) \in \mathbf{R}^{n+N}$, output $e(\cdot) \in \mathbf{R}$ and $\tilde{u}(\cdot) \in \mathbf{R}$.

The parameter $\alpha$ is defined by

$$
\alpha=\left(\alpha_{N}, \alpha_{N-1}, \ldots, \alpha_{2}, \alpha_{1}\right)^{T}
$$

and $F_{1}, F_{2}, F_{3}$ in (9) are identified by:

$$
\begin{aligned}
& F_{2}=\left(\begin{array}{lllll}
0 & 0 & \cdots & 0 & 1
\end{array}\right)^{T} ; \quad F_{3}=-\alpha^{T} ; \\
& F_{1}=\left(\begin{array}{ccccc}
0 & 1 & 0 & \cdots & 0 \\
0 & 0 & 1 & \cdots & 0 \\
\vdots & \vdots & \vdots & \vdots & \vdots \\
0 & \cdots & \cdots & 0 & 1 \\
-\alpha_{N} & \cdots & \cdots & -\alpha_{2} & -\alpha_{1}
\end{array}\right) \\
& =F_{0}-F_{2} \alpha^{T} \\
& F_{0}=\left(\begin{array}{ccccc}
0 & 1 & 0 & \cdots & 0 \\
0 & 0 & 1 & \cdots & 0 \\
\vdots & \vdots & \vdots & \vdots & \vdots \\
0 & \cdots & \cdots & 0 & 1 \\
0 & \cdots & \cdots & \cdots & 0
\end{array}\right)
\end{aligned}
$$

and

$$
\Psi(t)=\left(\begin{array}{c}
e(t-N) \\
e(t-N+1) \\
\vdots \\
e(-1)
\end{array}\right)
$$

where $\Psi(t)$ is built from the past values of tracking errors. $\tilde{x}(t), \tilde{u}(t), \tilde{y}(t)$ and $\tilde{e}(t)$ are constructed from transformed operation on the original plant as defined in (7) with:

$$
\begin{aligned}
\tilde{x}(t) & =P\left(\Delta^{-1}\right) x(t) \\
\tilde{u}(t) & =P\left(\Delta^{-1}\right) u(t) \\
\tilde{y}(t) & =P\left(\Delta^{-1}\right) y(t) \\
\tilde{e}(t) & =P\left(\Delta^{-1}\right)(r(t)-y(t))=-\tilde{y}(t)
\end{aligned}
$$

which states tracking errors as well as state vectors are both needed for $-N \leq t \leq-1$, they are arbitrary as far as the control input $u(t)$, $-N \leq t \leq-1$ is arbitrary.

As regard to the augmented state space representation (11), if the following two conditions in Lemma 1 are held, controllability and observability of (9) is guaranteed:

Lemma 1. (Owens et al.[16]) Given the new state-space representation (9), assume the original system with plant $G(z)$ is both controllable and observable. Under these conditions, the augmented state-space representation (9) is both controllable and observable if the following conditions are satisfied:

- The numerator of the original plant's transfer function $G(z)$, does not have any common factor with the polynomial:

$$
\begin{aligned}
\rho(z) & =z^{N} P\left(z^{-1}\right)=z^{N}+\alpha_{1} z^{N-1}+\cdots+\alpha_{N} \\
& =z^{N}+\Sigma_{j=0}^{N-1} z^{j} \alpha_{N-j} .
\end{aligned}
$$

- The original plant's transfer function $G(z)$ does not have any pole at $z=0$

There are a few classical control methods to find the stabilizing controller for the augmented system (9). Among these methods, optimal control is the one by solving the standard LQR problem on an infinite time interval $[0, \infty)$, which has to solve a minimization problem to minimize the following modified performance index (12). The selection of this performance index is inspired from a closely related area to optimal RC termed as optimal iterative learning control (ILC) [17]. The result is stated in Theorem 1 as below: 
Theorem 1. (Owens et al. [16]) Given a controllable and observable extended state-space representation (9), the state feedback solution of the optimal control problem obtained by minimizing

$$
J=\sum_{t=0}^{\infty}\left[e^{T}(t) Q e(t)+\tilde{u}^{T}(t) R \tilde{u}(t)\right]
$$

subject to the extended state dynamics, stabilizes the multi-periodic repetitive closed loop system in the sense that $\lim _{t \rightarrow \infty} e(t)=0$ and $\lim _{t \rightarrow \infty} \tilde{u}(t)=$ 0 .

As this is a classical infinity horizon linear quadratic regulation (LQR) problem with a state feedback solution, Theorem 1 can be easily proved. The constant feedback gain is then obtained by solving the well known discrete algebraic Riccati equation (DARE, [18]) for the solution

$$
K=\left(\begin{array}{ll}
K_{11} & K_{12} \\
K_{21} & K_{22}
\end{array}\right)
$$

Therefore, based on state matrices $\Pi, \Gamma, \Omega$ and weighting matrices $Q, R$, the optimal input $\tilde{u}(t)$ is given by:

$$
\begin{aligned}
\tilde{u}(t) & =-R^{-1}\left(\begin{array}{ll}
B^{T} & 0
\end{array}\right)\left(\begin{array}{ll}
K_{11} & K_{12} \\
K_{21} & K_{22}
\end{array}\right)\left(\begin{array}{l}
\tilde{x}(t) \\
\Psi(t)
\end{array}\right) \\
& =-R^{-1} B^{T} K_{11} \tilde{x}(t)-R^{-1} B^{T} K_{12} \Psi(t)
\end{aligned}
$$

Since $\tilde{u}(t)=P\left(\Delta^{-1}\right) u(t)=u(t)+\sum_{j=1}^{N} \alpha_{j} u(t-$ $j$ ), the actual form of multi-periodic repetitive controller is given by:

$$
\begin{aligned}
u(t)= & -\sum_{j=1}^{N} \alpha_{j} u(t-j)-R^{-1} B^{T} K_{11} \tilde{x}(t) \\
& -R^{-1} B^{T} K_{12} \Psi(t)
\end{aligned}
$$

As $t$ tends to infinity, both $\tilde{x}(t)$ and $\Psi(t)$ tend to zero. i.e. The control law ensures asymptotic zero tracking of the reference signal.

\subsection{A Problem of Dimensionality}

Although the structure of feedback controller (14) appears to be simple, it is computationally 'costly' to solve the high order DARE when the period of the reference signal is large. For instance, for a second order discrete time system, if the multi-periodic reference signal is formed by a linear combination of two periodic signals with periods 317 and 509 respectively, i.e. $n=2, N_{1}=$ 317 and $N_{2}=509$. From equation (9), order of the new state-space representation is $n+N_{1}+N_{2}$, i.e. $828 \times 828$. In order to find the optimal control input $\tilde{u}(k)$, high computational effort is inevitably required for solving DARE. Under these situations, computational efficient methods must be sought to deal with these high order tracking problems.

The problem mentioned above shows that the method proposed in [16] involves large computational problem as tracking reference signals contain large periods, so its applicability is very much restricted only to signals with small periods. To resolve this restriction, next we will propose a new method, where the main difference with the previous method given in [16] is that, the new one can be applied to track periodic and multiperiodic signals even when large periods present in reference signals, and the original high computational cost required by [16] can be largely reduced in many tracking problems by choosing different number of dominating poles.

\section{Low order controller design for discrete time linear MPRC systems}

\subsection{Motivation}

In Section 2, it is shown that an exact 'annihilating polynomial' of (5) can assure asymptotic zero tracking error. i.e. $e(t) \rightarrow 0$ as $t$ goes to infinity. However, as mentioned in the end of Section 2, computational problem occurs when individual periods are large. One of the remedies here is to turn to frequency analysis. In practical situations, transfer function $G(z)$ of the original system (1) is often finite dimensional and the reference signal $r(t)$ may also be concentrated to some frequencies rather than the whole band up to the Nyquist frequency, both $G(z)$ and $r(t)$ have limited bandwidth. Therefore, instead of applying the original full order "annihilating polynomial" $P\left(\Delta^{-1}\right)$ which contains all the frequency contents, these bandwidth properties give the designer an opportunity to reduce the dimensionality problem by designing a lower order 'annihilating polynomial', the following work is thus motivated for this purpose. 
As the internal model for MPRC systems, zeros of the "annihilating polynomial" $P\left(\Delta^{-1}\right)$ are evenly spread on the unit circle corresponding to the frequency elements of a multi-periodic signal. So the starting point will be based on spectral analysis of the reference signal $r(t)$ in frequency domain, where DFT (Discrete Fourier transform) analysis, which is computed by FFT (Fast Fourier transform), is to be applied.

\subsection{Frequency analysis of a multi-periodic signal}

First of all, as defined earlier, $r(t)$ is a multiperiodic reference signal in the form of (3) over $(-\infty,+\infty)$, with the relation between individual periods $N_{j}$ and sum of these periods $N$ is held by equation (4).

Periods $N_{j}$ considered in this paper are to be relatively prime, because the multi-periodic reference signal is usually not a periodic signal over one period of each periodic component ${ }^{1}$. In order to apply frequency analysis on $r(t), r(t)$ must be considered over a period of $N_{r}$, where $N_{r}$ is the least common multiple (LCM) of each individual period $N_{j}$ defined by:

$$
\begin{array}{r}
N_{r}=L C M\left(N_{1}, N_{2}, \cdots, N_{j}\right) \\
1 \leq j \leq M
\end{array}
$$

So the overall multi-periodic signal $r(t)$ is still a periodic one over the period $N_{r}$.

Given $v=\frac{2 \pi}{N_{r}}$ as the fundamental frequency for $r(t)$, it is well known that any periodic signal $r(t)$ can be written in terms of an exponential Fourier series representation:

$$
r(t)=\sum_{n v=-\infty}^{\infty} R(n) e^{j n v t}
$$

where $R(n)$ denotes DFT coefficient for each frequency over $(-\infty,+\infty)$. However, as explained earlier, many reference signals are often confined to only a certain frequency band $\omega_{d} \in\left[\omega_{l}, \omega_{u}\right]$ (the lower bound $\omega_{l}$ might be zero, but the upper bound $\omega_{u}$ cannot be infinity) but not whole frequency band up to Nyquist frequency; And in some cases, some frequency components have much dominated influences in certain selected frequencies than the others over the whole frequency region. In these situations, frequency analysis on $r(t)$ can often be separated into different groups depending on their dominance.

Assume $r(t)$ is represented by a sum of the dominating multi-periodic signal $r_{d}(t)$ and a non-dominating subordinate multi-periodic signal $r_{s}(t)$, where $r_{s}(t)$ is assumed to be so small that it can be ignored in controller design. Mathematically, it could be represented as:

$$
\begin{aligned}
r(t) & =\sum_{|n v| \in[-\infty, \infty]} R(n) e^{j n v t} \\
& =\underbrace{\left(\sum_{|n v| \in \omega_{d}} R(n) e^{j n v t}\right)}_{\text {Dominating } r_{d}(t)}+\underbrace{\left(\sum_{|n v| \notin \omega_{d}} R(n) e^{j n v t}\right)}_{\text {Non-dominating } r_{s}(t)}
\end{aligned}
$$

The basis of controller design depends on the following two principles:

1. The effect of $r_{s}(t)$ is ignored. Given an approximate low order "annihilating polynomial" $P_{r}\left(\Delta^{-1}\right)$, design is based on poles of $r_{d}(t)$, i.e. $P_{r}\left(\triangle^{-1}\right) r_{d}(t)=0$. By ignoring the effect of $r_{s}(t)$, $P_{r}\left(\Delta^{-1}\right) r(t) \approx 0$.

2. The effect of $r_{s}(t)$ on closed loop performance is checked afterwards.

To determine the dominating part $r_{d}(t)$, FFT analysis of a multi-periodic reference $r(t)$ will be defined as below:

Definition 1. For a given discrete time multiperiodic reference signal $r(t)$ defined by (3), where its individual periods are $N_{j},(1 \leq j \leq M)$, sum of periods is $N$, and $L C M$ is $N_{r}$ defined by (15), then $N_{r}$ point DFT analysis of $r(t)$ is given by:

$$
\begin{array}{r}
R(n)=\sum_{t=1}^{t=N_{r}} r(t) e^{-j 2 \pi(n-1) \frac{(t-1)}{N_{r}}} \\
1 \leq n \leq N_{r}
\end{array}
$$

and its inverse DFT is given by

$$
\begin{array}{r}
r(t)=\frac{1}{N_{r}} \sum_{n=1}^{n=N_{r}} R(n) e^{j 2 \pi(n-1) \frac{(t-1)}{N_{r}}} \\
1 \leq t \leq N_{r}
\end{array}
$$

\footnotetext{
${ }^{1}$ It might become a single period signal if periods $N_{j}$ are not co-prime
} 
If $\vec{R}_{m}$ is denoted as a vector of length $N_{r}$ with its elements as:

$$
\vec{R}_{m}=\left[R(1), R(2), \cdots, R\left(N_{r}\right)\right]^{T}
$$

By re-ordering the elements in (20), a newly sorted vector $\vec{S}_{m}$ in its descending magnitude value can be reshaped as follows:

$$
\vec{S}_{m}=\left[S(1), S(2), \cdots, S\left(N_{r}\right)\right]^{T}
$$

where $|S(1)| \geq|S(2)| \geq \cdots \geq\left|S\left(N_{r}\right)\right|$.

Equation (18) to (21) is a method to re-order the frequency contents of reference $r(t)$ in terms of their magnitude value over a period of $N_{r}$. However, it is generally known that a signal is not only decided by its magnitude value alone, but also its strength value, which is its energy.

\subsection{Energy analysis of a multi-periodic signal}

Mathematically, for a multi-periodic reference signal $r(t)$ containing individual periods $N_{j}, 1 \leq j \leq$ $M$. ( $N_{r}$ is the LCM of $\left.N_{j}\right)$, energy function of $r(t)$ over the LCM $N_{r}$ is denoted as below:

$$
E=\sum_{t=0}^{N_{r}-1}[r(t)]^{2}
$$

Parseval's theorem states that the total energy contained in a waveform $r(t)$ summed across all of time $t$ is equal to the total energy of the waveform's Fourier Transform $R(n)$ summed across all of its frequency components from $[-\pi,+\pi]\left(\mathrm{Or} N_{r}\right.$ points on the unit circle). Therefore, the following relation is held:

$$
\begin{aligned}
E=\sum_{t=0}^{N_{r}-1}[r(t)]^{2} & =\frac{1}{N_{r}} \sum_{n=0}^{N_{r}-1}|R(n)|^{2} \\
& =\frac{1}{N_{r}} \sum_{n=0}^{N_{r}-1}|S(n)|^{2}
\end{aligned}
$$

If $m$ number of poles are selected from the vector $\vec{S}$ in (21), then the energy content $E_{m}$ corresponding to these poles can be represented as:

$$
E_{m}=\frac{1}{N_{r}} \sum_{i=0}^{m}|S(i)|^{2}, \quad 0 \leq i \leq m
$$

In order to scale the percentage of $E_{m}(24)$ over the overall $E$ in equation (23). $\eta_{m}$ is defined as such a measurement variable, where

$$
\eta_{m}=\frac{E_{m}}{E}
$$

Define $\delta=1-\eta_{m}, \delta>0$, which is the energy difference. For $0 \leq m \leq N_{r}-1$, if $1-\eta_{m} \leq \delta$, i.e. $\eta_{m}$ is very close to 1 , then only $m$ number of poles will be selected to construct a low order "annihilating polynomial", which will act as an approximate $m$ order controller model. This controller will be applied to the original tracking problem instead of the exact model (5), it is expected to result in an approximate but not perfect tracking.

By combining the analysis made in Section 3.2 and Section 3.3, an algorithm is to be set up below for constructing a new low order "annihilating polynomial" model $P_{r}\left(\Delta^{-1}\right)$.

Remark 1. The other method for energy analysis of a multi-periodic reference signal can be carried out by applying DFT to each of its periodic component, in which case each component can also be separated into a dominating $r_{i d}(t)$ and nondominating part $r_{i s}(t)$, and select the dominating part for each periodic component $r_{i}(t)$. The difference between these two methods is that in the first method developed in this section, $0<m \leq N_{r}$, while for the second method, $0<m \leq N$. However, to compare the tracking performance in this paper with that of [16] and from DFT analysis result of $r(t)$, it is shown that only a maximum of $N$ number dominating poles from $N_{r}$ is enough for achieving perfect tracking. i.e. $\eta_{N}=E_{N} / E=1$. This also accords to the idea of Owens et al. [16] that selecting a total number of $N$ poles yield perfect tracking. So from now on, $m: \in[0, N]$.

\subsection{A low order multi-periodic repetitive controller design}

Following the analysis from Section 3.2 and 3.3, the main principle of this new method is that, when a required small energy difference tolerance $\delta$ is defined, only frequencies so that $1-\eta_{m} \geq \delta$ 
are selected to construct a low order "annihilating polynomial" $P_{r}\left(\triangle^{-1}\right)$, those frequencies with $1-\eta_{m}<\delta$ are ignored first for design purposes. The algorithm is summarized as follows:

Algorithm 1. A Low Order "Annihilating Polynomial" for Discrete Time Multi Periodic Systems.

Given a reference signal $r(t)$ defined by (3) with its individual periods as $N_{j}, 1 \leq j \leq M$ and the sum of period $N$ defined by (4). $N_{r}$ is $L C M$ of $N_{j}$ defined by (15).

1. Apply DFT analysis of $r(t)$ over a period of $N_{r}$ to obtain Fourier coefficient vector $\vec{R}_{m}$ with elements as (20). $0 \leq n \leq N_{r}-1$;

2. Re-order these elements in (20) by their magnitude value to form a new vector $\vec{S}$ with its elements re-arranged by their descending magnitude value as in (21);

3. Define the whole signal energy $E$ (23) of a reference signal over period $N_{r}$ and calculate energy content $E_{m}$ by (24) for $0 \leq m \leq N_{r}-1$ of the sorted new vector $\vec{S}$;

4. Define a tolerance $\delta>0$ and calculate $\eta_{m}$ defined by (25) for $0 \leq m \leq N-1$; (Remark 1)

5. When $1-\eta_{m} \leq \delta$, then only $m$ number of poles are chosen to construct a new low order "annihilating polynomial" with its z transform as

$$
\begin{aligned}
P_{r}\left(z^{-1}\right) & =\Pi_{q \in \omega}\left(1-z^{-1} e^{j \frac{2 \pi}{N_{r}} q}\right) \\
& =1+\sum_{i=1}^{m} \gamma_{i} z^{-i}
\end{aligned}
$$

where $\omega$ : frequency contents of $E_{m}$, and $m$ poles are chosen so that $1-\frac{E_{m-1}}{E}<\delta$ and $1-\frac{E_{m}}{E} \geq \delta$.

Remark 2. For the tracking/servo problem, in addition to the requirement of good matching between input and output, it is also known that at least one integrator is needed for robust tracking purposes. Meanwhile, since poles for a full order internal (or annihilating) model $P\left(z^{-1}\right)$ always come in pairs and are conjugate to each other, mathematically, if $\lambda_{k}=e^{j \frac{2 \pi}{N_{r}} k}$, then $\bar{\lambda}_{k}=$ $e^{j \frac{2 \pi}{N_{r}}\left(N_{r}-k\right)}=e^{-j \frac{2 \pi}{N_{r}} k}$, and integrator can be represented by $\frac{1}{1-z^{-1}}$, the more accurate form of the low order "annihilating polynomial" can be written as:

$$
P_{r}\left(z^{-1}\right)=\frac{1}{1-z^{-1}} \prod_{k=1}^{\frac{m-1}{2}} \mathcal{P}_{k} m \geq 3
$$

where $\mathcal{P}_{k}=\left(1-z^{-1} \lambda\right)\left(1-z^{-1} \bar{\lambda}\right)=1-2 z^{-1} h_{k}+$ $z^{-2}, h_{k}=\frac{\lambda+\bar{\lambda}}{2}=\cos \left(\frac{2 \pi}{N_{r}} k\right)$.

Remark 3. Unlike the work [16], this low order model may not always yield perfect tracking for multi-periodic signals containing more complex frequency contents, such as rectangular and square signals. However, when a designated $\eta_{m}$ or $\delta$ is given, this lower order model can be applied for tracking purposes even when the reference signal contains periods of very large value.

After designing such a low order "annihilating polynomial" $P_{r}\left(\triangle^{-1}\right)$, take a very similar procedure to that of $[16]$, the original tracking problem between control $u(t)$ and output $y(t)$ is converted into a non-standard LQR problem between a transfomed reduced controller $\tilde{u}_{r}(t)$ and error $e(t)$. Due to their similarities, the derivation procedure will be omitted. By the end of this derivation, a similar augmented state-space description to (9) is established. The actually form of such a description is to be given in (33), with the difference to (9) being that:

1. Extra terms $F_{2} P_{r}\left(\Delta^{-1}\right) r(t)$ and $P_{r}\left(\Delta^{-1}\right) r(t)$ are added to the benchmark solution in [16];

2 . The order of the augmented description is reduced from $n+N$ to $n+m$, where $n$ is the order of original discrete time system;

3. $N$ and $\alpha$ in state matrices are replaced by $m$ and $\gamma$ respectively.

In particular

$$
\Phi(t)=\left(\begin{array}{c}
e(t-m) \\
e(t-m+1) \\
\vdots \\
e(t-1)
\end{array}\right)
$$

An approximate multi-periodic $\mathrm{RC}$ controller is therefore formed as follows:

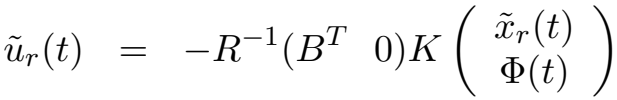

$$
\begin{aligned}
& =-R^{-1} B^{T}\left(K_{11} \tilde{x}_{r}(t)+K_{12} \Phi(t)\right) \\
& =-R^{-1} B^{T} K_{11} \tilde{x}_{r}(t)-R^{-1} B^{T} K_{12} \Phi(t)
\end{aligned}
$$


where $K$ is the optimal gain obtained from solving the low order DARE.

If $F_{r}$ and $\bar{G}_{r}$ are defined as $F_{r}=-R^{-1} B^{T} K_{11}$, $\bar{G}_{r}=-R^{-1} B^{T} K_{12}$ respectively, (29) changes to:

$$
\tilde{u}_{r}(t)=F_{r} \tilde{x}_{r}(t)+\bar{G}_{r}\left(\begin{array}{c}
e(t-m) \\
e(t-m+1) \\
\vdots \\
e(t-1)
\end{array}\right)
$$

Take $z$ transform on (30), which yields

$$
u(z)=F_{r} x(z)+T_{r}(z) e(z)
$$

where $T_{r}$ is represented by:

$$
T_{r}(z)=\frac{1}{P_{r}\left(z^{-1}\right)} \bar{G}_{r}\left(\begin{array}{c}
z^{-m} \\
z^{-m+1} \\
\vdots \\
z^{-1}
\end{array}\right)
$$

So the state feedback $F_{r} x(z)$ is functioning as a 'conditioning' term of the plant to make the 'classical error feedback' term $T(z) e(z)$ provide the required tracking performance. A block diagram of such a MPRC control system is provided in Figure 2 , where the approximate internal model $\frac{1}{P_{r}\left(z^{-1}\right)}$ in Figure 3 is included in $T_{r}(z)$.

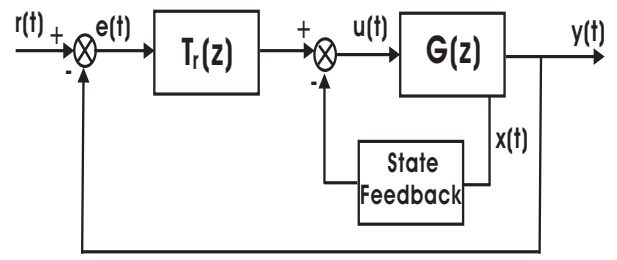

Figure 2. A block diagram of the low order controller based MPRC system

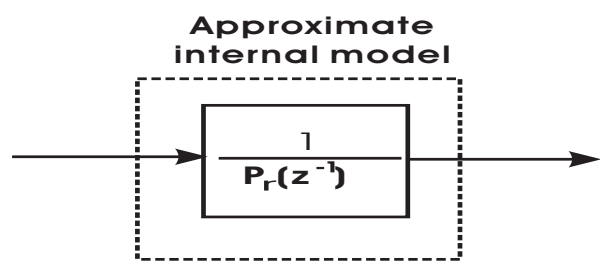

Figure 3. The low order internal model inside $T_{r}(z)$
Remark 4. In the above procedure, the algorithm design for a low order controller is aimed at tracking multi-periodic reference signals only. Nevertheless, this method could also be used when the reference signal contains only a single period, with the only difference being that, when the frequency analysis of a reference signal is considered, the least common multiple $N_{r}$ for a multi-periodic reference signal is now to be replaced by $N$, where $N$ is the period. Therefore, our earlier work [13] for tracking single period signals is also a special case for multi-periodic cases.

\subsection{Pole number choice vs tracking accuracy}

From equation (26) and (27), it is known that as more dominating poles are selected from (21), the closer of the low order "annihilating polynomial" gets to its full order model $P\left(\Delta^{-1}\right)$, and tracking results get more accurate. When $m=N$, $P_{r}\left(\Delta^{-1}\right)=P\left(\Delta^{-1}\right)$, asymptotic perfect tracking is gained. However, choosing more poles also reflect larger demand of energy content $\eta_{m}$ (or smaller value of $\delta_{m}$ ), which also require more computational effort. So there is always a tradeoff between pole number choice and tracking accuracy, which can be summarized as follows:

(1) Good tracking accuracy requires choosing more dominating poles, while

(2) Low computational effort needs choosing less dominating poles, so a lower order "annihilating polynomial" can be designed.

These two points will be shown more intuitively from the application examples in Section 4.

\subsection{BIBO stability analysis}

In this section, stability checking of closed loop multi-periodic repetitive systems will be briefly discussed. The result is summarized in Theorem 2 below:

Theorem 2. For a discrete time system (1), if the reference signal $r(t)$ is a multi-periodic signal including several periodic components each having large periods $N_{i}, 1<i<M$, and the reduced "annihilating polynomial" $P_{r}\left(\triangle^{-1}\right)$ of order $m$ is obtained from Algorithm 1. Assume the original system plant $G(z)$ is both controllable and observable, then the tracking error $e(t)$ is bounded if $P_{r}\left(\triangle^{-1}\right) r(t)$ is bounded. i.e. The closed loop system meets BIBO stability. 
Proof. This proof is separated into two steps.

Step 1: Assume $P_{r}\left(\triangle^{-1}\right)$ is a complete "annihilating polynomail" of reference signal $r(t)$, i.e. $P_{r}\left(\triangle^{-1}\right) r(t)=0,-\infty<t<\infty$. In this situation, proof is exactly matching to Appendix A of Owens' work in [16]. Therefore details of this part is omitted for brevity.

By applying the derivation process in [16], an augmented state-space representation resembling a close match of (9) is derived, where $\tilde{u}(t)=P_{r}\left(\triangle^{-1}\right) u(t)$, the initial condition $\Phi(0)=$ $\{e(-m), e(-m+1), \cdots, e(-1)\}^{T}$, states are given by $\tilde{x}(t)=P_{r}\left(\triangle^{-1}\right) x(t)$ and those ones defined in (28).

Then from Theorem 1 in [16], the closed loop repetitive system is asymptotically stable in the sense of $\lim _{t \rightarrow \infty} e(t)=0$ and $\lim _{t \rightarrow \infty} \tilde{u}(t)=0$, which means that all poles of the closed loop system are inside the unit circle.

Step 2: If $P_{r}\left(\triangle^{-1}\right)$ is not a full order "annihilating polynomial", i.e. $P_{r}\left(\triangle^{-1}\right) r(t) \neq 0,-\infty<$ $t<\infty$. Under this circumstance, derivation of an augmented state-space representation is based on the earlier assumption $P_{r}\left(\triangle^{-1}\right) r(t) \approx 0$. The difference being that, by the end of the derivation, this augmented state-space description is a modified form of (9) but with the similar matrix forms and states as Step 1, where it has the following form:

$$
\begin{aligned}
Z(t+1) & =\Pi_{r} Z(t)+\Gamma_{r} \tilde{u}_{r}(t)+F_{2 r} P_{r}\left(\Delta^{-1}\right) r(t) \\
e(t) & =\Omega_{r} Z(t)+P_{r}\left(\Delta^{-1}\right) r(t)
\end{aligned}
$$

$\Pi_{r}, \Gamma_{r}, F_{2 r}$ and $\Omega_{r}$ have the similar forms as their earlier defined counterparts $\Pi, \Gamma, F_{2}$ and $\Omega$ except for $N$ and $\alpha$ in the latter matrices are now replace by $m$ and $\gamma$ respectively. All the definitions are the same as those in Step 1. From (33), it is also possible to write error $e(t)$ as follows:

$$
\begin{aligned}
e(t)= & \Omega_{r}\left(z I-\Pi_{r}+R^{-1} \Gamma_{r} K\right)^{-1} F_{2 r} P_{r}\left(\Delta^{-1}\right) r(t) \\
& +P_{r}\left(\Delta^{-1}\right) r(t)
\end{aligned}
$$

From step 1, if reference signal $r(t)$ could be written as $r(t)=r_{d}(t)+\left(r(t)-r_{d}(t)\right)$, where $r_{d}(t)$ is the collection of dominating part of reference signal $r(t)$ that gives $P_{r}\left(\Delta^{-1}\right) r_{d}(t)=0$. Since $\Omega_{r}\left(z I-\Pi_{r}+R^{-1} \Gamma_{r} K\right)^{-1}$ has all the poles inside the unit circle, it is asymptotic stable. In addition to this, as $P_{r}\left(\Delta^{-1}\right)\left(r(t)-r_{d}(t)\right)$ is bounded, the closed loop tracking error is bounded.

Remark 5. Although analysis is only considered for reference tracking only, it can be applied straightforwardly to multi-periodic disturbance rejection with minor modifications. For a multiperiodic disturbance signal $d(t)$, if it is added to the input signal $u(t)$, the system representation is then formed as:

$$
\begin{aligned}
x(t+1) & =A x(t)+B(u(t)+d(t)), x(0)=x_{o} \\
y(t) & =C x(t)
\end{aligned}
$$

Remark 6. For a system including both reference tracking and disturbance rejection, if both reference and disturbance signals contain periodic components of same or different periods $N_{j}$, the low order controller scheme developed in this paper can also be applied. The signals can be any arbitrary ones as long as they are of periodic nature.

\section{Application to A Physical Model}

The experimental test-bed has previously been used in a number of publications $[15,19]$ to evaluate $\mathrm{RC}$ related algorithms. The non-minimum phase mass damper spring system contains a rotary mechanical system of inertias, dampers, torsional springs, a timing belt, pulleys and gears. Figure 4 shows the non-minimum phase characteristic of this test-bed, where $\theta_{i}$ and $\theta_{o}$ are the input and output positions, $J_{1}$ and $J_{g}$ are inertias, $B$ is a damper, $K$ is a spring and $G$ represents the gearing. A further spring mass-damper system as in Figure 5 is connected to the input in order to increase the relative degree and complexity of the system.

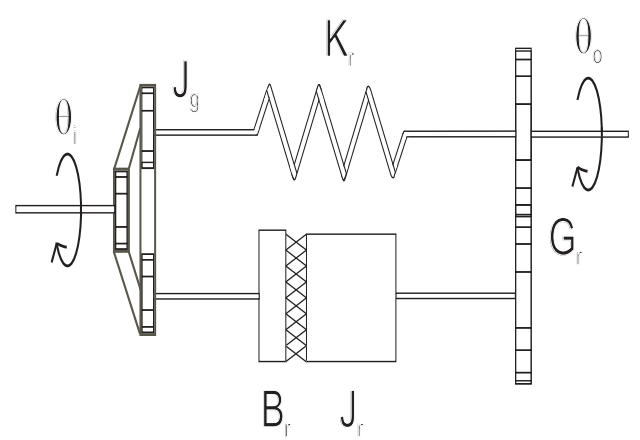

Figure 4. Non-minimum phase characteristic 


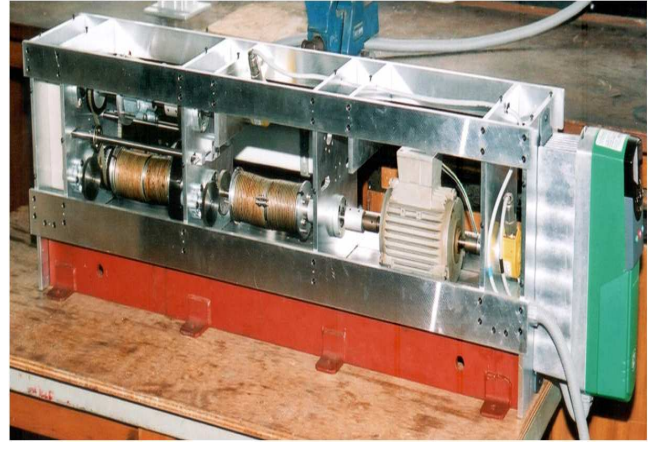

Figure 5. Experimental test-bed

The system is modeled using a least-meansquares (LMS) algorithm to fit a linear model to a great number of frequency response test results [19], where the continuous system is given as follows:

$$
y(t)=\frac{1.202(4-s)}{s(s+9)\left(s^{2}+12 s+56.25\right)} u(t)
$$

A PID loop is used around the plant to produce superior results. The PID gains are $K_{p}=$ $137, K_{i}=5$ and $K_{d}=3$. The system is sampled using a zero order hold with a sampling frequency of $h=25 \mathrm{~Hz}$, i.e. sampling time $T=$ $\frac{1}{25}=0.04 \mathrm{sec}$, the discretized (or sampled) model for closed loop feedback system of (35) and PID loop is established having a state-space representation in the form of (1), where $A, B, C$ are as follows:

$$
\begin{gathered}
A=\left(\begin{array}{ccccc}
4.2351 & -1.7861 & 1.5012 & -0.6295 & 0.4224 \\
4 & 0 & 0 & 0 & 0 \\
0 & 1 & 0 & 0 & 0 \\
0 & 0 & 1 & 0 & 0 \\
0 & 0 & 0 & 0.25 & 0
\end{array}\right) \\
B=\left(\begin{array}{llllll}
0.0625 & 0 & 0 & 0 & 0
\end{array}\right)^{T} \\
C=\left(\begin{array}{llllll}
0.0543 & 0.0150 & 0.0180 & -0.0225 & 0.0119
\end{array}\right)
\end{gathered}
$$

As $\left(\begin{array}{ll}A^{T} & B\end{array}\right)$ is controllable and $\left(C^{T} \quad A^{T}\right)$ is observable, the augmented state space representation obtained in the form of (33) by ignoring terms including $P_{r}\left(\Delta^{-1}\right) r(t)$ is also controllable and observable (Lemma 1). For simplicity, all the experimental work carried out in this section will assume zero initial conditions, i.e. $x_{0}=0$.

\subsection{Tracking a single periodic reference}

The first experiment is to track a reference signal that contains only a single period. As indicated in Remark 4, although Algorithm 1 is designed for multi-periodic tracking circumstances, it can easily be applied to single period tracking cases by setting $N=N_{1}=N_{r}$. The continuous reference signal is $r(t)=\sin \left(\frac{2 \pi}{3} t\right)$ of period $3 \mathrm{sec}, t \in(-\infty,+\infty)$, ( $\mathrm{t}$ is continuous). By choosing the sampling frequency as $h=25 \mathrm{~Hz}$, the sampled reference signal is $r(t)=\sin \left(\frac{2 \pi}{75} t\right)$, $1 \leq t<+\infty$ ( $\mathrm{t}$ is a digital time), with sample number $N=75$. Weighting matrices are chosen as $R=1$ and $Q=I$.

Figure 6 displays a pole-zero map for the full order "annihilating polynomial" in its $z$ transformation as $P\left(z^{-1}\right)=1-z^{-75}$, where all 75 poles evenly spread on the unit circle. Here $\eta_{i}=0.9999$, i.e. $\delta=10^{-4}$, to meet the condition $1-\eta_{i} \geq \delta$, Figure 7 shows that instead of choosing all 75 poles, only $m=3$ poles inside the circle are required to be chosen. i.e. poles at $\lambda_{0}=1, \lambda_{1}=$ $0.9965-0.0837 i$ and $\bar{\lambda}_{1}=0.9965+0.0837 i$ are selected, where the first pole indicates the integrator and the other two indicate the fundamental frequency of reference signal, in a conjugate pair. Therefore, the low order "annihilating polynomial" is designed as:

$$
\begin{aligned}
P_{r}\left(z^{-1}\right) & =\left(1-z^{-1}\right)\left(1-\lambda_{1} z^{-1}\right)\left(1-\bar{\lambda}_{1} z^{-1}\right) \\
& =1-2.9930 z^{-1}+2.9930 z^{-2}-z^{-3}
\end{aligned}
$$

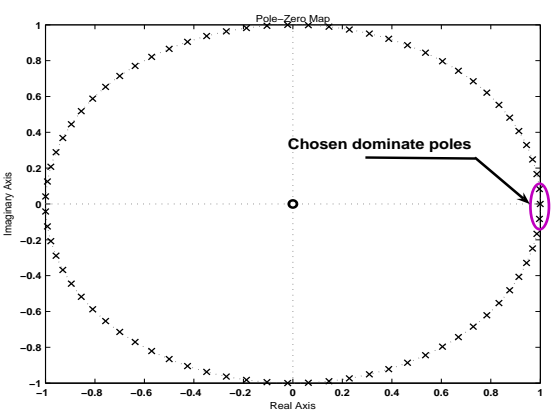

Figure 6. Pole-zero map of $\frac{1}{P\left(\Delta^{-1}\right)}$ 


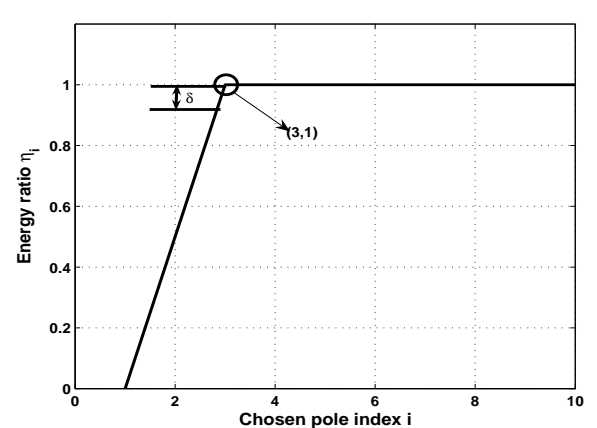

Figure 7. Energy ratio $\eta_{i}$ over chosen pole index $i$

The approximate optimal low order controller (26), for which only a 5 order DARE is required to be solved, is thus written by the following form:

$$
u(z)=F_{r} x(z)+T_{r}(z) e(z)
$$

where:

$$
\begin{aligned}
& F_{r}=\left[\begin{array}{lllll}
-39.6586 & 7.5295 & -20.4157 & 27.0414 & -17.1418
\end{array}\right] \\
& G_{r}=\left[\begin{array}{lll}
0.8982 & -1.8903 & 1.0000
\end{array}\right]
\end{aligned}
$$

and the forward path transfer function is:

$$
T_{r}(z)=\frac{898.2-1890 z^{-1}+1000 z^{-2}}{1-2.979 z^{-1}+2.965 z^{-2}-0.986 z^{-3}}
$$

Figure 8 shows the tracking error $e(t)$ and controller $u(t)$ considered over a time of $t \in$ $[0,20]$ sec. The results show that as time gets larger, near perfect tracking is achieved with low control effort.
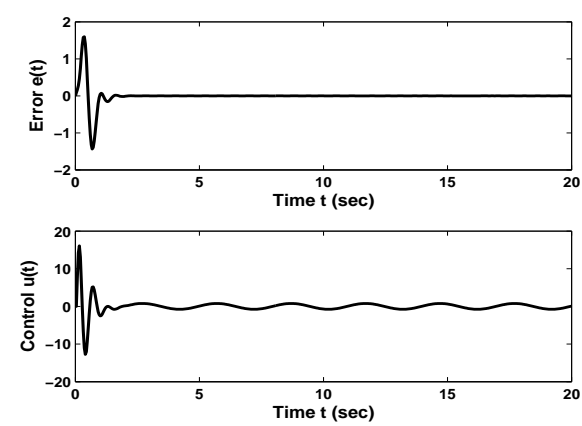

Figure 8. Tracking error e(t) and control u(t) for Sinusoidal Signal

Alternatively, Figure 9 also shows the logarithm error norm $\|e(k)\|$ over a 'cycle' number period $N$, where $\|e(k)\|)$ is defined as follows:

$$
\|e(k)\|=\sqrt{\frac{\sum_{t=k}^{k+N-1} e^{2}(t)}{N}}
$$

and $t$ is a digital time index.

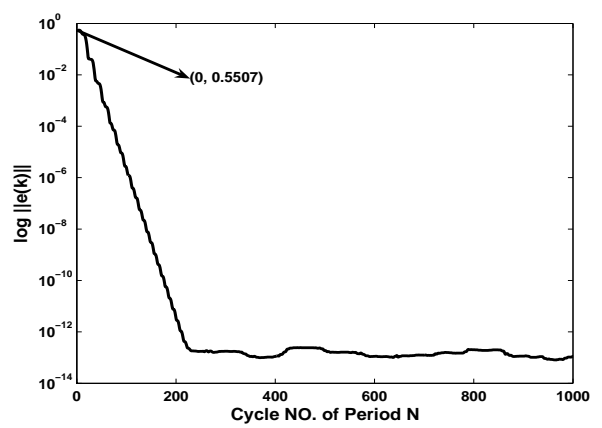

Figure 9. Logarithm plot of Error Norm $\|e(k)\|$

Figure 9 also indicates that the new low order controller effectively tracks the reference signal $r(t)$ and drive error $e(t)$ to near zero value.

\subsection{Tracking multi-periodic signals}

In order to test the low order multi-periodic repetitive controller on more general multi-periodic signals, and to excite more frequency contents, in the second experiment, tracking is focused on a more complex multi-periodic reference signal - signals containing triangular periodic components. Assume the same experimental test-bed is used but the reference signal contains two triangular periodic components with periods $T_{1}=0.92 \mathrm{sec}$ and $T_{2}=1.16 \mathrm{sec}$ respectively. A plot of $r(t)$ is given in Figure 10 over a time interval $t \in[0,30] \mathrm{sec}$.

For simplicity, sampling frequency of $h=25 \mathrm{~Hz}$ is re-used, by which it gives $N_{1}=23$ and $N_{2}=29$. The LCM of $N_{1}$ and $N_{2}$ is $N_{r}=677$. Weighting matrices $Q$ and $R$ are again chosen as $Q=$ $I$, and $R=1$. The energy measurement value is designated as $\eta_{m}=0.9998$, i.e. tolerance value is $\delta=0.0002$.

As the multi-periodic reference signal becomes a single periodic signal over $N_{r}$, the complete "annihilating polynomial" should be $P\left(z^{-1}\right)=$ $1-z^{-677}$, or by the method introduced in [16], it should at least have an order of $P\left(z^{-1}\right)=$ $\left(1-z^{-23}\right)\left(1-z^{-29}\right)$. 


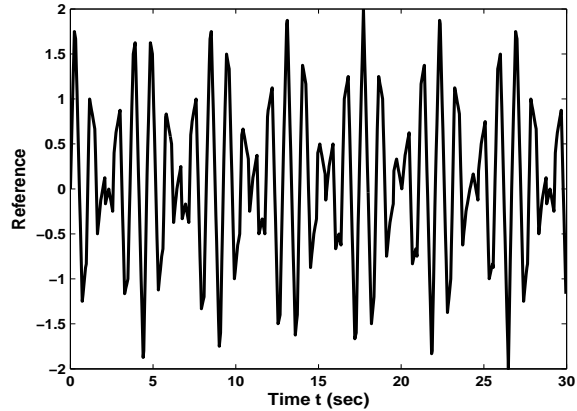

Figure 10. A reference with two triangular signals

Figure 11 is a pole-zero map for $\frac{1}{P\left(z^{-1}\right)}=$ $\frac{1}{1-z^{-677}}$, given $\delta=0.0002$, Figure 12 indicates that only $m=39$ poles need to be selected, so that $1-\eta_{i} \approx \delta$. These chosen pole positions are listed in Table. 1 that are numbered counter-clockwise from 0 to 676 , where position 0 represents the pole that intersects with positive real axis.

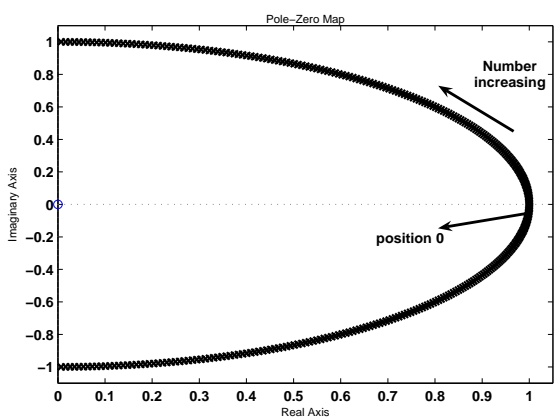

Figure 11. Pole-zero map for $\frac{1}{P\left(z^{-1}\right)}$

Table 1. Poles selected from pzmap Figure 11

\begin{tabular}{|c|c|c|c|c|c|c|}
\hline 638 & 29 & 644 & 23 & 0 & 69 & 598 \\
\hline 87 & 580 & 621 & 46 & 92 & 575 & 522 \\
\hline 145 & 609 & 58 & 203 & 464 & 115 & 552 \\
\hline 138 & 529 & 483 & 184 & 116 & 551 & 406 \\
\hline 261 & 230 & 437 & 319 & 348 & 506 & 161 \\
\hline 232 & 435 & 493 & 174 & & & \\
\hline
\end{tabular}

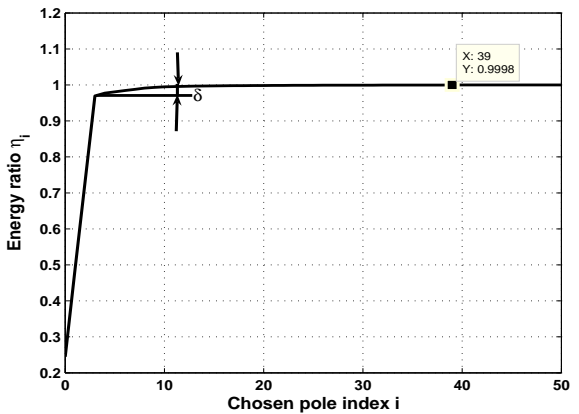

Figure 12. Energy Ratio Over Poles Choice

Based on these analysis, the low order "annihilating polynomial" with an order of $m=39$ is designed and the low order controller can be obtained by only solving a DARE of order 39, where it bears its form as (31).
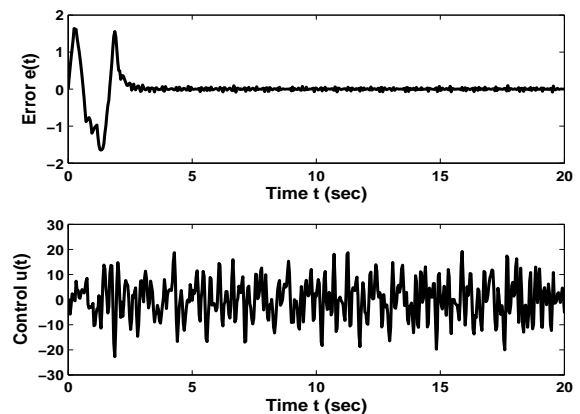

Figure 13. Error $\mathrm{e}(\mathrm{t})$ and Control $\mathrm{u}(\mathrm{t})$

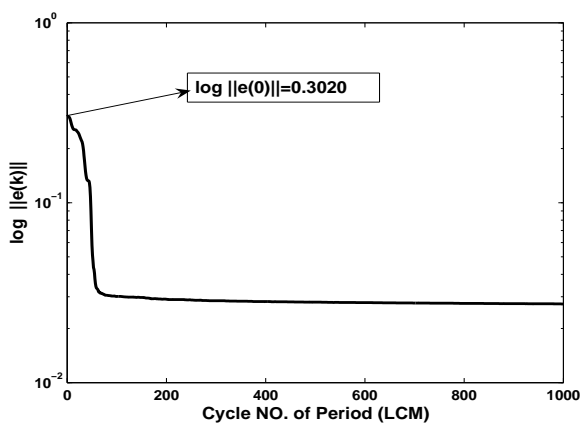

Figure 14. Error norm of e(k) over cycle NO. $\mathrm{k}$ of period (LCM)

As expected, Figure 13 shows that error $e(t)$ is small and bounded as $t \rightarrow \infty$, and only a small control effort $u(t)$ is required for achieving such a purpose, where tracking $99.98 \%$ energy contents of reference signal $r(t)$ is achieved. Again, Figure 14 also shows logarithm plot of error norm $\|e(k)\|$ to the 'cycle' No. of period $N$, the result indicates bounded tracking error $\|e(k)\|$ smaller than $10^{-1}$. 


\subsection{Impact of poles number choice on tracking performance}

In this part, the impact of different number of poles selection from $\vec{S}$ on ultimate tracking performance is discussed. For simplicity, the above multi-periodic reference signal is tracked but different number of poles are chosen. The results are shown in Figure 15.

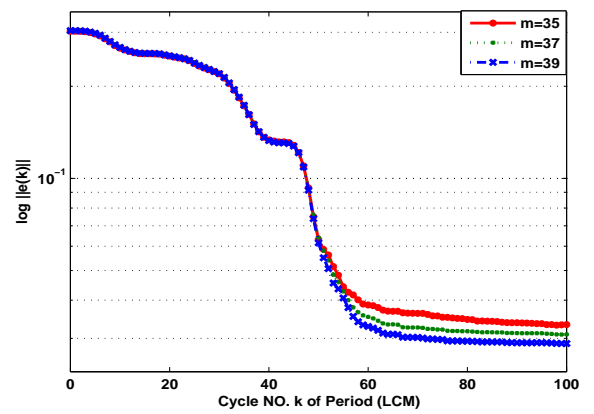

Figure 15. Comparison results of $\|e(k)\|$ as poles number $m$ is different

From Figure 15, it is seen that as the number of dominating poles is selected as $\mathrm{m}=35,37$ and 39 respectively, the logarithm error norm $\log \|e(k)\|$ decreases, i.e. the more dominating energy contents are chosen, the more accurate tracking result is. Therefore, when $m=N_{1}+N_{2}=52$ (apply an exact model), it is expected to give an asymptotic perfect tracking, which matches with the previous work given in [16].

\section{Conclusions and Future Research}

In this paper, a new low order controller scheme is developed for tracking problems in optimal MPRC systems, where the high order DARE causes the difficulty of designing a proper stabilizing controller.

For solving this problem, frequency analysis of reference signal is carried out, by which the magnitude value is compared and re-ordered over the frequency domain up to the Nyquist frequency. Then, energy analysis on the multi-periodic reference signal is carried out to choose the dominating poles but disregard the non-dominating ones, which depends on the requirement of different tracking accuracy designated by $\eta_{m}$ or an energy tolerance $\delta$. By doing so, a low order "annihilating polynomial" $P_{r}\left(\Delta^{-1}\right)$ is designed by Algorithm 1 and a corresponding low order MPRC law is formed that can be applied to track any arbitrary periodic signals with known large periods for different tracking accuracy purposes. The designed low order control law also reduces the original high computational requirement posed by solving high order DARE and it also gives bounded tracking error. To assess the effectiveness of this controller, it is applied to a mass damper spring system and experiments are carried out on tracking both single and multi-periodic reference signals, and both on a simple sinusoidal signal and the one that contains triangular signal components. Experimental results demonstrate that very accurate tracking results are gained for both experiments. Meanwhile, the effect of pole number choice on tracking performance is also discussed, where as more dominating poles are selected, tracking result gets better.

Future work can be carried out in the following areas.

1. Due to some practical reasons, in this paper, only the model of a physical system is used to illustrate validity of the new low order controller. It is therefore recommended to give an experimental validation in the near future.

2. Robust analysis. Due to the fact that the designed low order controller scheme includes a state-feedback term and the effect of a forward element, as seen in Figure 2, it is necessary to analyze the effect of the state-feedback and forward terms on tracking performance.

3. The implementation of low order control law still requires using an observer, even though this will not affect the ultimate stability and tracking accuracy, it does affect transient performance. This can be an open area.

4. Uncertainty of periods. Although the work is concentrated for tracking reference signals with already known periods, in some cases, periods are not known in advance. So it is necessary to extend the current research to those cases, in which case adaptive control can be applied to RC/MPRC systems.

5. In both [16] and this work, the optimality method is applied to find the stabilizing control law. However, given the augmented statespace representations (9) and (33), the stabilizing controller could be designed by methods other than optimization, where the control law is not an optimal one but nor does it need to solve the DARE. Therefore, the computation problem can 
also be solved. Comparisons between these methods can be discussed based on their tracking performances.

\section{Acknowledgments}

Youde Han was supported by a studentship from the Department of Automatic Control and Systems Engineering, University of Sheffield.

\section{References}

[1] Francis, B.A. and Wonham, W.M., The internal model principle for linear multivariable regulators, Applied Mathematics and $\mathrm{Op}$ timization, 2(1), 170-194, (1975).

[2] Garimella, S.S. and Srinivasan, K., Application of repetitive control to eccentricity compensation in rolling, Journal of Dynamic Systems Measurement and Control 118,(1996)

[3] Tomizuka, M., Tsao, T.C., and Chew, K.K., Analysis and Synthesis of DiscreteTime Repetitive Controllers, Journal of Dynamic Systems, Measurement and Control. (1989)

[4] Sadegh, N., and Guglielmo, K. (1995), "A new repetitive controller for mechanical manipulators," Journal of Robotic Systems, Copyright (c) 1991 Wiley Periodicals, Inc. A Wiley Company.

[5] Yamada, M., Riadh, Z., and Funahashi, Y. (2000), "Discrete Time Repetitive Control System with Multiple Periods," IEEE.

[6] Manayathara, T.J., Tsao, T.C., Bentsman, J., and Ross, D. (1996), "Rejection of Unknown Periodic Load Disturbances in Continuous Steel Casting Process Using Learning Repetitive Control Approach," IEEE Trans. On Control Systems Technology, 4, 259-265.

[7] Yamada, M., Funahashi, Y., Ishihara, S., and Matsushita, M. (1996), "Extended DiscreteTime Prototype Repetitive Controllers and Its Application," Proceedings of the 35th Conference on Decision and Control, Kobe, Japan. IEEE.

[8] Sato, H., Sueno, T., Toyama, T., Mikawa, M., and Toda, T. (1991), "High accuracy Magnet Power Supply for Proton Synchrotron By Repetitive Control," IEEE.

[9] Ghosh, J., and Paden, B. (2000), "Nonlinear repetitive control," IEEE Transactions, Automatic Control.
[10] Horowitz, K.K. (1990), "Repetitive and adaptive control of robot manipulators with velocity estimation," IEEE Trans on Robotics and Automation, 13, 204-217.

[11] Ahn, H.S. (2003), Discrete Time Adaptive Repetitive Control and Its Application to a Linear BLDC Motor, The Fourth International Conference on Control and Automation (ICCA'03), Montreal, Canada.

[12] Chotai, A. and Owens D.H., Digital control design using reduced order models, in Proceedings of the IEE International Conference Control85 (1985)

[13] Han, Y., Owens, D.H. and Chu, B, Discrete Time Linear Optimal Repetitive Control A Low Order Controller Scheme, Proceedings of the 2010 2nd International Conference on Computational Intelligence, Communication Systems and Networks, Livepool, UK, 238 243, (2010)

[14] Hillerstrom, G., and Sternby, J., "Repetitive Control Using Low Order Models," American Control Conference, Volume: 2, On page(s): 1873- 1878. (1994)

[15] Wang, L., Chai, S., Freeman, C.T., and Rogers E., On Structure Selection for Multivariable Repetitive-predictive Controllers, 49th IEEE Conference on Decision and Control December, Hilton Atlanta Hotel, Atlanta, GA, USA, 15-17, (2010)

[16] Owens, D.H., Marian., T.R., Hatonen, J.J. and Li, L.M., Discrete time linear optimal multi-periodic repetitive control: a benchmark tracking solution, International Journal of Control, 79:9, 991-1001, (2006)

[17] Amann, N., Owens, D.H. and Rogers, E., Iterative learning control using optimal feedback and feedforward actions, International Journal of Control, 65, 277-293, (1996)

[18] Astrom, K.J. and Wittenmark, B., Computer Controlled Systems: Theory and Design, 2nd ed., Englewood Cliffs, NJ: Prentice Hall (1990).

[19] Freeman, C.T., Lewin, P.L., Hätönen, J., Owens, D.H., and Ylinen, R. , An optimality based Repetitive Control algorithm for discrete-time systems, IEEE, Transactions on Circuits and Systems-I: Regular Papers, 55, No.1. (2008) 
Youde Han received both his Master and Ph.D degrees from the Department of Automatic Control and Systems Engineering, University of Sheffield, United Kingdom in 2004 and 2010 respectively. Title of his Ph.D thesis is "Issues of optimality and computation in repetitive control". Presently he is a Post-doctor researcher in Mechatronic Engineering Department, ATILIM University, Ankara, Turkey. His current research is focused on "mimicking of Human Motor Control with bio-inspired approaches on Robot Arms"

David H. Owens was the Head of the Department of Automatic Control and Systems Engineering from 1999-2008 and Dean of Engineering at Sheffield University in the period 2002-2006. He was the Convener of the Deans in the period 2005-06. Prior to this he was Head of the School of Engineering and Computer Science at the University of Exeter where he also acted as Chairman of Exeter Enterprises Ltd. He was the elected Chairman of the United Kingdom Automatic Control Council from 1999-2002 representing the interests of the UK Professional Institutions within IFAC. He was also elected as a Fellow of the Royal Academy of Engineering in 2008 in recognition of his contributions to control theory and practice and the profession over the past four decades. He is currently an Emeritus Professor at Sheffield University. He is the author of over 500 refereed technical publications and the author or co-author of four books.

Bing Chu received his Bachelor and Master from Tsinghua University, Beijing, China during September 2000 to January 200\%. After that, he received his Ph.D degree from Department of Automatic Control and Systems Engineering, University of Sheffield, United Kingdom in December 2009. Between February 2010 C May 2010, he worked as a Research Scientist at Iterate Control Ltd. in Sheffield. From June 2010 to date, he is working as a Post Doctor researcher at University of Oxford, United Kingdom. His research specialties include Iterative learning control; Multidimensional systems; Nonlinear filtering; Repetitive control; Spatially distributed dynamic systems. 